\title{
SAFETY OF ACUTE COMBINED PHYSICAL EXERCISE SESSION IN PATIENTS WITH TAKAYASU ARTERITIS: A PILOT MULTICENTER RANDOMIZED CLINICAL STUDY
}

Alexandre Moura dos Santos ${ }^{1, *}$, Rafael Giovani Misse ${ }^{1}$, Isabela Bruna Pires Borges ${ }^{1}$, Alexandre Wagner Silva de Souza ${ }^{2}$, Rosa Maria R Pereira ${ }^{1}$, Samuel Katsuyuki Shinjo ${ }^{1}$

1. Universidade de São Paulo, São Paulo (SP), Brazil. 2. Universidade Federal de São Paulo, São Paulo (SP), Brazil.

*Corresponding author: alexandrestos@usp.br

\section{BACKGROUND}

Physical exercise has shown to be an important adjuvant tool in comorbidity treatment and improving the quality of life in several systemic autoimmune diseases. However, existing studies have not assessed the safety of the acute combined physical exercise (CPE) session in patients with Takayasu arteritis (TAK).

\section{MATERIALS AND METHODS}

This multicenter randomized clinical study was carried out from 2019 to 2020, where 14 adult patients with TAK were randomized (1:1) into two groups: patients who were submitted (TAKS) or not (TAKR) to an acute CPE session (involving a 5-minutes treadmill warm-up followed by moderate-intensity strength exercises: bench press, bench squat, cable seated row, and leg press at $45^{\circ}$, as well as three sets of 10-12 repetitions and rest interval between 45 and 60 seconds). The following parameters were also evaluated at baseline and after the CPE session for the first 2 hours and, then, for 1 month: C-reactive protein, erythrocyte sedimentation rate, disease activity (by ITAS2010 and NIH questionnaires), and adverse effects (e.g., dizziness, dyspnea, headache, high blood pressure, arrhythmia, and general pain).

\section{RESULTS}

The TAKR and TAKS groups had comparable age [40.8 (40.0-46.0) vs. 39.7 (34.8-48.8) years, respectively], body mass index [28.9 (22.5-28.9) kg/m2 vs. $27.3(26.1-29.9) \mathrm{kg} / \mathrm{m} 2$ ], and duration of disease [16.0 (9.0-20.0) vs. 17.0 (12.0-21.0) years], all p > 0.05. In each group, comparing the baseline versus after intervention data, the C-reactive protein and erythrocyte sedimentation rate values did not alter $(p>0.999)$ (Figure 1). There were also no adverse events or disease relapses.

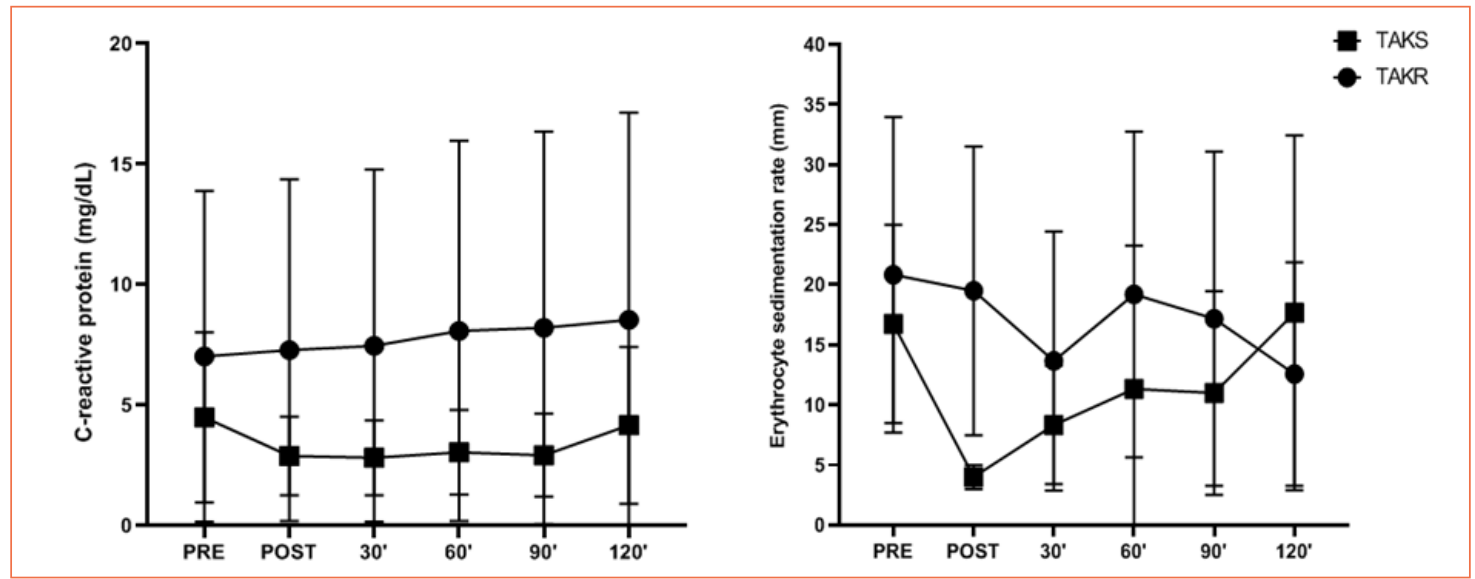

Figure 1. Kinetics of protein C-reactive and erythrocyte sedimentation rate at baseline and after intervention in the patients with Takayasu arteritis. Patients submitted (TAKS) or not submitted (TAKR) to an acute combined physical exercise. 


\section{CONCLUSION}

A CPE session is safe to use on patients with TAK, and did not produce adverse effects or disease relapses.

\section{FUNDING}

Fundação de Amparo à Pesquisa do Estado de São Paulo (FAPESP Grant No. 2018/08735-3 to AMS; 2019/12155-5 to RGM; 2019/11367-9 to IBPB; and 2019/11776-6 to SKS). 